\title{
Enfrentando la desigualdad y la corrupción: Agencia, empoderamiento y desarrollo democrático
}

\author{
DAVID A. CROCKER \\ University of Maryland (Estados Unidos de América) \\ dcrocker@umd.edu
}

\begin{abstract}
Resumen
En este trabajo identifico cuatro temas que, considero, deberían ser más prominentes en el enfoque de las capacidades para el desarrollo internacional y la reflexión ética respecto de los fines, medios y procesos del desarrollo: (1) desigualdad de poder, (2) agencia y empoderamiento, (3) democracia y desarrollo y (4) corrupción. Sostengo que el primero y el cuarto son desafíos de urgencia para la campaña del enfoque de las capacidades, y que el segundo y tercer temas son importantes maneras en las cuales los desafíos pueden y deberían ser enfrentados.
\end{abstract}

Palabras claves: Desigualdad, corrupción, democracia, justicia global.

\section{Confronting inequality and corruption: Agency, empowerment, and democratic development}

\begin{abstract}
In this paper I identify four topics that I argue should be more prominent in the capability approach to international development and in ethical reflection on the ends, means, and processes of development: (1) inequality of power, (2) agency and empowerment, (3) democracy and development, and (4) corruption. I argue that the first and fourth are urgent challenges for the capability approach ad the second and third topics are important ways in which the challenges can and should be met.
\end{abstract}

Key words: Inequality, corruption, democracy, global justice.

Doctorado de la Universidad de Yale (PhD, M.Div y MA), Estados Unidos, Actualmente se desempeña como Senior Research Scholar en la Escuela de Políticas Públicas en la Universidad de Maryland. Fue fundador y anterior presidente de la Asociación Internacional de Ética del Desarrollo (IDEA). Actualmente es director del programa de especialización en Desarrollo Internacional en la Universidad de Maryland. Autor del libro Ethics of Global Development: Agency, Capability, and Deliberative Democracy (2008).

Traducción de Gabriela Arriagada Bruneau.

Recibido: 4/Enero/2016 - Aceptado: 29/Enero/2016 
¿Cuáles son los temas que el enfoque de las capacidades y la ética del desarrollo deberían enfatizar y vincular en los años venideros? ${ }^{1}$ Creo que dentro de los problemas apremiantes que han de tratarse están los siguientes: (1) desigualdad de poder, (2) agencia y empoderamiento, (3) democracia y desarrollo y (4) corrupción. El primero y el último son desafíos para los cuales el segundo y tercero son, al menos, parte de la solución.

\section{Desigualdad de poder}

La desigualdad de poder y su "precio" para un (buen) desarrollo, desde lo local a lo global, se ha convertido en un discutido tema alrededor del mundo. Stiglitz (2012, 2013, 2014), Piketty (2013) y Krugman (2014) son solo tres nombres asociados con argumentos que se encargan de algún tipo de desigualdad y que dicen que esta es la causa y consecuencia no solo de un desarrollo imperfecto, sino que también de una mala gobernación ${ }^{2}$. Dados los compromisos éticos que tienen, los especialistas en ética del desarrollo deben dedicarse a producir el mejor trabajo posible en economía política, si es que han de lidiar éticamente con la privación y transformación humanas. Economistas políticos se preguntan cómo y por qué el poder, de varios tipos, es distribuido dentro de una unidad social. ¿Cuánta desigualdad existe en el poder económico, político y so-

1 Este ensayo está basado en el artículo "Agencia, Empoderamiento y Desarrollo Democrático", el cual expuse en la conferencia de la Asociación de Desarrollo Humano y Capacidades llevada a cabo en Atenas (Grecia) en Septiembre del 2014. Además, realicé una versión más temprana y más larga como el orador principal en el décimo Congreso Internacional y décimo tercer aniversario de la Asociación Internacional de Ética del Desarrollo en San José, Costa Rica en Julio del 2014. Ese artículo, "Development and Global Ethics: Five Foci for the Future" apareció en The Journal of Global Ethics, 10(3), 2014: 1-8. Estoy muy agradecido con muchos de mis colegas quienes han contribuido a lo largo de los años a mis visiones constantemente en evolución, respecto de la naturaleza y misión democrática y enfocada en el agente que tiene la ética del desarrollo y la ética global. Por sus útiles comentarios a este artículo agradezco a Edna Crocker, Larry Crocker, Jay Drydyk, Sirkku Hellsten, Eric Palmer, Matthew Regan, Marie Clarie Vasquez Durán y Engda Wubneh.

2 Para un resumen accesible, véase Doyle \& Stiglitz (2014). Stiglitz de una manera más técnica trata la naturaleza, causas y consecuencias de varios tipos de desigualdad en Stiglitz (2012; 2013). Véase Piketty, 2013; Krugman 2014a. Para evidencia de que las élites «extractivas» son la principal causa del fracaso de una nación, y que la «inclusividad» económica y política es la solución básica, véase también Acemoglu \& Robinson (2012) Un problema con las consideraciones de Acemoglu \& Robinson es que ellos no logran clarificar de manera suficiente si es que este gobierno «inclusivo» es «democrático» y, si es que lo es, qué clase de gobierno democrático tiene mayor legitimidad en diversos contextos. 
cial? ¿Cuáles son las causas y relaciones de estas desigualdades y sus consecuencias para un buen desarrollo en cualquier nivel? ¿Cuáles son las maneras más efectivas y duraderas para reducir las desigualdades? Complementando estas preguntas empíricas, el experto en ética del desarrollo se pregunta: ¿qué, si es que hay algo, está mal con los varios tipos de desigualdad? ¿qué, si es que hay algo, debería hacerse con estas desigualdades, quién o qué debería hacerlo, y quién debería decidir? Respecto a la gobernanza, por ejemplo, el experto en ética evalúa lo que Michael Johnston describe como "la discusión política sobre preguntas de quién ha de gobernar a quién, bajo qué derecho, a través de qué medios, y dentro de qué límites" (Johnston, 2014: 2; ver también Forst, 2014).

Es importante, por supuesto, preguntar "¿Desigualdad de qué?" y considerar no solo las desigualdades de ingresos y riquezas, sino también desigualdades horizontales (entre grupos religiosos y étnicos), desigualdades de libertades que las personas tienen razones para valorar, y desigualdades en la agencia, incluyendo agencia política. Y, para la mayoría de los expertos en ética del desarrollo y ética global, el crecimiento económico no es el fin del desarrollo, sino que es solamente un medio. Pero incluso si es que definimos desigualdad en base a ingresos y/o riquezas, y desarrollo en base al crecimiento económico, "desigualdad" como Paul Kraugman lo plantea es una "carga". El economista político y columnista del The New York Times no rehúye de las implicancias éticas y políticas del nuevo igualitarismo:

¿Acaso no es cierto que cobrar impuestos a los ricos y ayudar a los pobres reduce el incentivo de ganar dinero? Bueno, sí, pero los incentivos no son lo único que importa para el crecimiento económico. La oportunidad también es algo crucial. Y la desigualdad extrema priva a muchas personas de tener la oportunidad para alcanzar su potencial. Piénselo. ¿'Tienen los niños norteamericanos que son talentosos pero de familias con bajos ingresos, las mismas oportunidades de utilizar su talento - para tener una educación adecuada, para continuar con una carrera profesional adecuada- que tienen los niños que nacen más arriba en la escalera? Por supuesto que no. Es más, esto no es solamente injusto, tiene además un precio muy alto. La desigualdad extrema significa un desperdicio de recursos humanos (Krugman, 2014b).

Así como economistas políticos y columnistas de opinión no se abstienen de realizar juicios éticos y prescripciones políticas, los expertos en ética del desarrollo deben basarse en el trabajo y las controversias más creíbles en economía política. Amartya Sen, un economista-filósofo quien se toma las causas y consecuencias económicas y políticas muy seriamente, ha hecho de su trabajo normativo y político con Jean Drèze 
en India (Drèze \& Sen, 1999, 2002, 2013) cada vez más persuasivo, ahondando progresivamente en el análisis de los desbalances de poder en India y el "agarre de la desigualdad" (Drèze \& Sen, 2013). Lo que se requiere no son valores puros o hechos puros, sino un enfoque multidisciplinario en el cual los valores proveen una manera de ver y evaluar los hechos en cuanto hechos, sus causas y efectos, permitiéndonos entender y explicar lo que es, ha sido, es probable que sea y lo que podría ser -si los agentes actúan apropiadamente (Crocker, 2008; véase Gasper, 2012). Sin un ancla en la economía política, el enfoque de las capacidades ${ }^{3}$ y la ética del desarrollo arriesgan un entendimiento inadecuado de las limitaciones y las oportunidades para políticas y acciones éticamente justificadas.

\section{Agencia y empoderamiento}

La agencia y el empoderamiento se han vuelto importantes ideales en la reflexión ética sobre el desarrollo y la justicia global, pero los expertos en ética deberían distinguirlos entendiendo a la agencia como necesaria pero insuficiente para aquello que queremos decir con empoderamiento (Drydyk, 2013). Los expertos en ética están en deuda con Sen por su distinción realizada en 1985, entre agencia y bienestar (Sen, 1985; véase Crocker \& Robeyns, 2009). Los actores del desarrollo y las instituciones deberían respetar, apoyar y restaurar ambos aspectos de la existencia humana, pero la agencia y el bienestar, aunque usualmente relacionados, son diferentes. Una persona o grupo es un agente, en la medida que esa persona o grupo decide y actúa con un propósito y por un propósito deliberado, sin coacciones externas o internas, haciendo una diferencia en el mundo. En ese caso, la persona o grupo es auto-determinada, el autor de su propia vida, en vez de un objeto de la coacción de otro o víctima de las circunstancias (Crocker 2008, 153-63; Crocker \& Robeyns 2009). La vida de una persona va bien, cuando sus "ser y hacer" son tales que la persona tiene razones para valorarlos, y se da cuenta de esto en su vida o tiene la libertad de darse cuenta de esta posibilidad. El estar bien alimentado, sano, seguro y feliz son ejemplos de un bienestar alcanzado; tener oportunidades reales o libertades para "ser y hacer" son ejemplos de libertades de bienestar. Para ejercer la libertad de agencia (y tener logros a partir de esta agencia), el agente requiere, al menos, un bienestar mínimo; y

3 En inglés "capability approach", implica la importancia de alcanzar un bienestar, moralmente hablando; y la libertad de alcanzar este bienestar está asociada a las capacidades de cada persona. Véase Robeyns (2011) (N. del T.). 
mediante el ejercicio de esta agencia, el agente tiene la posibilidad de incrementar su bienestar.

Hasta el momento, todo bien. Pero, sin embargo, es cada vez más evidente no solo que los agentes pueden ejercer su agencia en maneras que reducen e incluso acaban con su propio bienestar, sino que además, en maneras que agreden o terminan con el bienestar de otros. Una manera de entender el ideal de empoderamiento, entonces, es que individuos y grupos empoderados son agentes que tienen el poder en cuanto agentes, tienen la capacidad de trabajar en pos de su bienestar y el de otros, y hacerlo luchando y superando las desigualdades de poder que limitan su agencia y bienestar (Drydyk, 2013). Una mujer no está empoderada si ella es meramente libre de conseguir empleo fuera de casa, puesto que los poderes fácticos pueden someterla a trabajos caracterizados por acoso, discriminación y un bienestar reducido (Koggel, 2013). Las mujeres están empoderadas en la medida que, individual y colectivamente luchan contra y (algunas veces, al menos) superan el despliegue de poderes en contra de su agencia y bienestar (Koggel, 2013). Se sigue que las DE y GE deben preocuparse no solo de promover la agencia, sino también el protagonismo moralmente permisible que decide, desafía, combate y busca superar los obstáculos sociales para una mejor vida (Drydyk, 2013). La economía política, como ha sido expuesta anteriormente, delinea y expone esos obstáculos, especialmente aquellos relacionados a varias desigualdades.

\section{Democracia y desarrollo}

El desarrollo y la democracia han de ser integrados en la teoría y la práctica del desarrollo en todos los niveles. Muchos de los expertos en ética y profesionales han argumentado - persuasivamente, creo yo- que los ciudadanos afectados por proyectos de desarrollo local deberían tener un rol en la toma de decisiones en todas las fases de esos proyectos. Estas fases incluyen la identificación de necesidades, deliberación sobre fines y medios, monitoreo y evaluación. Las personas tienen un derecho a ejercer su agencia y ayudar a determinar y modificar las políticas bajo las cuales viven. El hacer esto da a los participantes una sensación de que son agentes respetados y que ellos "poseen" sus proyectos. Una consecuencia es que la democracia local -especialmente cuando es inclusiva y provee una oportunidad a las voces que están marginadas a no ser oídasreduce el peligro del apresamiento de las élites y contribuye a la durabili-

4 DE y GE son abreviaciones en inglés de los términos Development Ethics y Global Ethics, a saber, Ética del Desarrollo y Ética Global, respectivamente (N. del T.).

VERITAS, No 34 (Marzo 2016) 
dad de un proyecto de desarrollo. Algunos importantes ejemplos incluyen el presupuesto municipal inclusivo (participativo) en Brasil y a lo largo de Latinoamérica, así como otros canales y lugares para que los ciudadanos ejerciten su agencia empoderada (Baiocchi et al, 2011; Cameron et al, 2012; Goldfrank, 2012; Vásquez Durán, 2014).

La democracia local, sin embargo, no es suficiente. Cada vez más, estudiosos defienden, y también profesionales están reconociendo, que la democracia local debe ser apoyada y, al mismo tiempo, ha de apoyar a la democracia nacional (así como también regional y global). Incluso las instituciones más innovadoras de democracia local pueden llegar a ser «administradas», socavadas o capturadas por poderosas élites económicas, éticas, políticas y religiosas. La democracia, en todos los niveles, requiere un desarrollo equitativo; y un desarrollo durable y centrado en el ser humano, una democracia amplia y profunda que haga una diferencia.

$\mathrm{Al}$ menos tres factores, que los expertos en ética deben exponer y criticar, dificultan la deseada integración del desarrollo -ya sea local, nacional o global- y la democracia. Primero, desde la Segunda Guerra Mundial, la asistencia para el desarrollo ha sucumbido, reiteradamente a la «tentación de lo técnico» evitando los problemas de gobernanza y, en un sentido amplio, la política (Carothers \& de Gramont, 2013). Definiendo el desarrollo como un crecimiento económico libre de valores, varios expertos en desarrollo concibieron que los esfuerzos de promover un desarrollo interno o que la ayuda para el desarrollo entregada a países en vías de este, son "objetivos" y técnicos, muy alejado de la guerra fría y la acrimonia política. Pero ahora, sabemos que la asistencia técnica, como uniones de crédito o mallas anti-malaria para camas, pueden reforzar regímenes represivos. La ayuda alimenticia para personas famélicas, por ejemplo, puede incrementar la hambruna cuando los precios que reciben los agricultores por sus cosechas deflactan, o puede causar que las naciones receptoras de esta ayuda fracasen en el ataque de la causa de esta hambruna. Los países receptores se vuelven dependientes de externos, y los proyectos de desarrollo fracasan por falta de propiedad ciudadana. Las élites son capaces de capturar asistencia políticamente "neutral" y fortalecer su dominancia mediante sistemas de patrocinio y corrupción. Cuando la ayuda para el desarrollo es exclusivamente económica, se ignoran "las aspiraciones más amplias de los ciudadanos, que van más allá del éxito económico, como por ejemplo los deseos populares de una dignidad política y empoderamiento" (Carothers \& de Gramont, 2013: 5). El resultado, evidente en el norte de África, es el fracaso que la asistencia para el desarrollo genera, al no poder abocarse a las tensiones políticas, las cuales consecuentemente explotan. 
Un segundo obstáculo para la integración de la democracia y el desarrollo, es la tendencia de varias instituciones financieras internacionales y gobiernos nacionales de enfrentar los problemas mencionados anteriormente con una solución de sondeo neutral, una "buena gobernanza", en vez de una forma de democracia inclusiva y profunda. Y esa gobernanza, basada en el cumplimiento de la ley y la competencia electoral es compatible con una "democracia dirigida" o un "autoritarismo competitivo" (Levitsky \& Way, 2010). De hecho, el rival actual más serio de la integración para el desarrollo y la democracia es una nueva forma de autoritarismo "benevolente", en el cual se realizan elecciones -incluso aquellas razonablemente libres y justas- pero estas son artefactos mediante los cuales la élite se aferra al poder. Una de las razones por las que los negocios e intereses financieros chinos están en marcha hacia el mundo "desarrollado", es que los "desarrolladores" chinos -prometiendo dejar el autoritarismo local o la democracia deficiente intactos- proveen infraestructura a cambio de acceso a recursos y otros beneficios. Un ejemplo es que China ha trabajado en la construcción de un nuevo estadio nacional de fútbol para Costa Rica y, así también, ha acordado financiar y controlar la alternativa nicaragüense del Canal de Panamá.

La tentación autoritaria es grande. Una manera de desafiarla es apelando a la evidencia entregada por Acemoglu y Robinson que dice que las naciones en un mediano y largo plazo fracasan debido a la exclusividad económica y política (Acemoglu \& Robinson, 2012). Otra manera es que los expertos en ética del desarrollo y ética global expandan la noción de democracia, incluyendo en esta movimientos sociales y militantes democráticos que trabajen en pos de una igualdad de condiciones. En vez de identificar la democracia únicamente con "un ritual de una sola vez que sucede en el día de la elección", "la democracia es un trabajo de todo el año" (Kiai \& Vize, 2014) en el cual los ciudadanos, especialmente los grupos e individuos marginados, usan o crean una serie de canales y lugares mediante los cuales pueden gobernar (Deveaux, 2003; Kosko, 2013). Una repercusión a esto, es que la democracia y las sociedades democráticas deberían desvincular la ciudadanía de cualquier afiliación de identidad étnica o religiosa y afirmar un "sistema de gobierno de arco iris”, una ética pluralista (Kymlicka, 2007; Kosko, 2013; Muasher, 2014). Una objeción a este clase de pluralismo liberal y democrático es que los ciudadanos no podrán mantenerse leales e identificados con un sistema de gobierno a menos que esté enraizado en una (sola) etnia o religión. Para un argumento contrario, véase Anna Stiltz, Liberal Loyalty: Freedom, Obligation, and the State (Stiltz, 2011). 


\section{Corrupción}

La corrupción también debería ser abordada de manera más explícita y robusta en la DE y la GE. Los expertos en ética del desarrollo inicialmente rehuyeron de este asunto. ¿Por qué? Porque la primera cosa $-\mathrm{y}$, a veces, la única cosa- que llamaba la atención de un entrevistador en nuestro "campo" era nuestra "solución" a X corrupción (donde X es una persona o institución, ya sea gubernamental o no-gubernamental). Queriendo evitar el "apuntar con el dedo al otro" una fijación en la personalidad, en vez de tratar el tema de instituciones deficientes y políticas gubernamentales desde un punto de vista ético, los expertos en ética del desarrollo intentaron (la mayoría de las veces, insatisfactoriamente) evitar este tipo de preguntas y cambiar el tema. $\mathrm{Y}$ es interesante notar que -con una excepción (Penz, Drydyk \& Bose, 2011)- el término "corrupción" no aparece en los índices de grandes trabajos en la ética del desarrollo (Goulet, 1971, 1989; Nussbaum, 2001, 2011; Gasper, 2004; Dower, 2007; Crocker, 2008; Deneulin, 2014) ${ }^{6}$.

Es importante destacar que los expertos en ética del desarrollo no estaban solos en este fracaso de ocuparse de la corrupción. No fue sino hasta mediados de 1990 que el Banco Mundial comenzó a atacar este problema. ¿Por qué tardó tanto? La corrupción, obviamente, se ligó a los problemas de gobernanza y política. Hasta 1990 la visión del Banco era tener una misión únicamente económica y técnica y, en ningún modo, infringiendo en los asuntos internos del país. Sin embargo, el Banco, llegó a darse cuenta eventualmente, que la corrupción impedía los logros propios del Banco, los cuales respondían a metas de crecimiento económico para países en desarrollo.

La corrupción puede y debería ser el trabajo central de la DE y la GE, las cuales, como ya he dicho, se construyen a partir de los mejores

5 La expresión original utilizada en inglés es: "self-righteous finger-wacing". Debido al contexto la explicación es similar al uso en español de apuntar con el dedo a alguien, en este caso, a los expertos en ética (finger-waving) Mientras que "self-righteous" refiere a que este «apuntar con el dedo» viene desde una auto-justificación, es decir, un juicio propio de quien está realizando la acción o el enunciado ( $N$. del T.).

6 Expertos en ética del desarrollo, Peter Penz, Jay Drydyk y Pablo S. Bose, proponen una "integridad respecto de la corrupción" como uno de los siete valores para un desarrollo "que vale la pena" y distinguir, correctamente, una norma de integridad apropiada, clarificada y defendida, la cual tiene un importante rol en el combate contra la corrupción (Penz, Drydyk, \& Bose, 2011: 148-50). En su nuevo libro sobre India, An Uncertain Glory: India and Its Contradictions, Drèze \& Sen (2013) dedican un importante capítulo a la corrupción -sus causas y soluciones- y la responsabilidad. En mi futuro trabajo, analizaré y evaluaré el argumento de los autores en relación con la avalancha de trabajos recientes dedicados al tema de la corrupción. 
trabajos de economía política. Podría decirse, además, que un desarrollo defectuoso causa y es causado por la corrupción. Abordar el tema de la corrupción requiere de un trabajo teórico, análisis político, evaluación ética y un compromiso práctico.

El trabajo teórico incluye argumentaciones conceptuales, históricas y empíricas respecto de la naturaleza, tipos, causas, tenacidad y consecuencias de la corrupción tanto gubernamental como no-gubernamental. Los expertos en ética deberían hacerse cargo del mérito moral, la efectividad y la durabilidad de diferentes tipos de estrategias para atacar y reducir la corrupción.

La evaluación ética enfatiza aquello que es moralmente malo respecto de la corrupción y, así también, la cualidad moral (o falta de esta) de distintas estrategias anti-corrupción. Dicho de manera más simple, ¿cuáles son los derechos de los ciudadanos y los deberes de los Estados con respecto a las diversas formas de corrupción? (Johnston, 2014).

El trabajo político requiere la identificación de posibles maneras para mejorar las leyes anti-corrupción, los incentivos y las instituciones. El enfoque de las capacidades en conjunto con los estudiosos debe investigar el rol que juegan y deberían jugar la integridad personal, el compromiso moral (fuerza moral) y la educación cívica en el combate contra la corrupción.

Una de las maneras más prometedoras para enfrentar el problema de la corrupción es que los expertos en DE y GE se relacionen con el trabajo del cientista político Michael Johnston ${ }^{7}$. Dentro de sus publicaciones más importantes relacionadas con la corrupción están Syndromes of Corruption: Wealth, Power, and Democracy (Johnston, 2005) y Corruption, Contention, and Reform: The Power of Deep Democratization (Johnston, 2014; véase también Hoseah, 2008; Penz, Drydyk, \& Bose 2011; Rotstein, 2011; Kimeu, 2014). Johnston nos dice que la causa fundamental de la corrupción, la cual él define como "el abuso de roles públicos para beneficio privado" (Johnston, 2014: 9), es una especie de desequilibrio de poder. No obstante, él también establece que las constelaciones de poder y los tipos de corrupción varían significativamente y, además, que las estrategias para combatir la corrupción deben variar de acuerdo a los diferentes tipos de corrupción. Lo que funciona con un tipo de corrupción, puede fallar con otro tipo e incluso terminar creando mayor corrupción. Lo que tienen en

7 Véase también otros estudios recientes sobre corrupción, tales como el de Sarah Chayes, Thieves of State: Why Corruption Threatens Global Security (Chayes, 2015); Louise I. Shelley, Dirty Entanglements: Corruption, Crime and Terrorism (Shelley, 2014); Zephyr Teachout, Corruption in America: From Benjamin Franklin's Snuff Box to Citizens United (Teachout, 2014); y Frank Vogl, Waging War on Corruption: Inside the Movement Fighting the Abuse of Power (Vogl, 2012).

VERITAS, No 34 (Marzo 2016) 
común todas las estrategias anti-corrupción, que son efectivas y están éticamente justificadas, es lo que él denomina "democratización profunda" - no el construir una democracia en un sentido electoral o constitucional, sino que permitiendo y promoviendo a los ciudadanos el fiscalizar los abusos de riquezas y poder mediante una propugnación política de sus propios intereses (Johnston, 2014: xiii). Cada país acosado por la corrupción puede y debe enfrentar este problema de manera democrática, mediante una discusión pública a nivel nacional, con prensa crítica e independiente, regulaciones promulgadas democráticamente y una ciudadanía movilizada, motivada a defender sus intereses y, a ejercer su agencia empoderada.

\section{Conclusión}

Los cuatro temas interrelacionados que aquí he discutido, solo destacan los problemas que han estado implícitos, secundarios o emergentes, tanto en el enfoque de las capacidades como en la ética del desarrollo. Dado ciertos eventos recientes -desde Siria e Irak hasta Ferguson, Missouri- queda aún más claro que un buen desarrollo desde lo local a lo global es complejo y urgente. Con un nuevo, o al menos un énfasis más robusto para atacar los males de la desigualdad, autoritarismo y la corrupción y, además, un nuevo compromiso de avanzar en el empoderamiento ciudadano con una democracia inclusiva, tal enfoque de capacidades centrado en la agencia y la ética del desarrollo pueden seguir "manteniendo la esperanza" y contribuir al progreso de la humanidad.

\section{REFERENCIAS}

-Acemoglu, D. \& Robinson, J. A. (2012). Why Nations Fail. New York: Random House/Crown Business.

-Baiocchi, G., Heller, J. Heller \& Silva, M. (2011). Bootstrapping Democracy: Transforming Local Governance and Civil Society in Brazil. Stanford: Stanford University Press.

-Cameron, M. A., Herschberg, E. \& Sharp, K. E. (2012). New Institutions for Participatory Democracy in Latin America: Voice and Consequence. New York: Palgrave Macmillan.

-Carothers, T. \& de Gramont, D. (2013). Development Aid Confronts Politics: The Almost Revolution. Washington, D.C.: Carnegie Endowment for International Peace.

-Crocker, D. A. (2008). Ethics of Global Development: Agency, Capability, and Deliberative Democracy. Cambridge: Cambridge University Press.

-Crocker, D. A. \& Robeyns, I. (2009). Capability and Agency. En C. Morris (Ed.), Amartya Sen (pp. 60-90). Cambridge: Cambridge University Press. 
-Dower, N. (2014). Global Ethics: Dimensions and Prospects. Journal of Global Ethics, 10 (1), 8-15.

-Doyle, M. W. \& Stiglitz, J. E. (2014). Eliminating Extreme Inequality: A Sustainable Development Goal, 2015-2030. Ethics \& International Affairs, 28 (1), 5-13.

-Deveaux, M. (2003). A Deliberative Approach to Conflicts of Culture. Political Theory, 31 (6), 780-807.

-Drèze, J. \& Sen, A. (1999). India: Economic Development and Social Opportunity. Oxford: Oxford University Press.

-Drèze, J. \& Sen, A. (2002). India: Development and Participation (2nd Ed.) Oxford: Oxford University Press.

-Drèze, J. \& Sen, A. (2013). An Uncertain Glory: India and Its Contradictions. Princeton: Princeton University Press.

-Drydyk, J. (2013). Empowerment, Agency, and Power. Journal of Global Ethics, 9 (3), 249-262.

-Drydyk, J. (2014). Foundational Issues: How Must Global Ethics be Global? Journal of Global Ethics, 10 (1), 16-25.

-Forst, R. (2014). Justification and Critique: Towards a Critical Theory of Politics. Cambridge, UK and Malden, MA: Polity.

-Gasper, D. (2004). The Ethics of Development: From Economism to Human Developent. Edinburgh: Edinburgh University Press.

-Gasper, D. (2012). Development Ethics - Why? What? How? A Formulation of the Field. Journal of Global Ethics, 8 (1), 117-35.

-Goldfrank, B. (2012). Deepening Local Democracy in Latin America: Participation, Decentalization, and the Left. University Park: Penn State University Press.

-Goulet, D. (1971). The Cruel Choice: A New Concept in the Theory of Development. Cambridge: Atheneum.

-Goulet, D. (1989). Incentives for Development: The Key to Equity. New York: New Horizons Press.

-Johnston, M. (2005). Syndromes of Corruption: Wealth, Power, and Democracy. Cambridge and New York: Cambridge University Press.

-Johnston, M. (2014). Corruption, 2Contention, and Reform: The Power of Deep Democratization. Cambridge: Cambridge University Press.

-Kiai, M. \& Vize, J. (2014). Three Years after Tunisia: Thoughts and Perspectives on the Rights to Freedom of Assembly and Association from the United Nations Special Rapporteur Maina Kiai. Journal of Global Ethics, 10 (1), 114-121.

-Kimeu, S. (2014). Corruption as a Challenge to Global Ethics: The Role of Transparency International. Journal of Global Ethics, 10 (2), 231-37.

-Koggel, C. M. (2013). A Critical Analysis of Recent Work on Empowerment: Implications for Gender. Journal of Global Ethics, 9 (3), 263-75.

-Kosko, S. J. (2013). Agency Vulnerability, Participation, and the Selfdetermination of Indigenous Peoples. Journal of Global Ethics, 9 (3), 293-310. 
-Krugman, P. (2014a). Why We're in a New Gilded Age. Review of Piketty, Thomas. 2014. Capital in the Twenty-first Century. New York Review of Books, May 8.

-Krugman, P. (2014b). Inequality is a Drag. New York Times, August 7.

-Kymlicka, W. (2007). Multicultural Odysseys: Navigating the New International Politics of Diversity. New York: Oxford University Press.

-Levitsky, S. \& Way, L. A. (2010). Competitive Authoritarianism: Hybrid Regimes After the Cold War. Cambridge: Cambridge University Press.

-Muasher, M. (2014). The Second Arab Awakening: And the Battle for Pluralism. New Haven: Yale University Press.

-Nussbaum, M. (2000). Women and Human Development: The Capabilities Approach. Cambridge: Cambridge University Press.

-Nussbaum, M. (2011). Creating Capabilities: The Human Development Approach. Cambridge, MA: Harvard University Press.

-Penz, P., Drydyk, J. \& Bose, P. (2011). Displacement by Development: Ethics, Rights, and Responsibilities. Cambridge: Cambridge University Press.

-Piketty, T. (2014). Capital in the Twenty-first Century. Cambridge: Belknap Press.

-Robeyns, I. (2011). The Capability Approach. En E. N. Zalta (ed.), The Stanford Encyclopedia of Philosophy (Summer 2011 Edition), <http://plato.stanford.edu/archives/sum2011/entries/capabilityapproach/>

-Rothstein, B. (2011). The Quality of Government: The Political Economy of Corruption, Social Trust, and Inequality in an International Comparative Perspective. Chicago: University of Chicago Press.

-Sen, A. (1985). Well-being, Agency and Freedom: The Dewey Lectures 1984. Journal of Philosophy, 82 (4), 169-221.

-Sen, A. (1993). Capability and Well-being. En A. Sen y M. Nussbaum (Eds.), The Quality of Life (pp. 30-53). Oxford: Oxford University Press.

-Sen, A. (1999). Development as Freedom. New York: Anchor Books-Random House.

-Stiglitz, J. E. (2012). Macroeconomic Fluctuations, Inequality, and Human Development. Journal of Human Development and Capabilities, 13 (1), 31-58.

-Stiglitz, J. E. (2013). The Price of Inequality: How Today's Divided Society Endangers Our Future. New York: W. W. Norton.

-Stiglitz J. E. (2014). Eliminating Extreme Inequality: A Sustainable Development Goald, 2015-2030. Ethics \& International Affairs, 28 (1), 5-13.

-Stilz, A. (2011). Liberal Liberty: Freedom: Freedom, Obligation, and the State. Princeton: Princeton University Press.

-Vasquez Durán, M. C. (2014). Participatory Budgeting in the Dominican Republic: Implications for Agency, Democracy and Development. Ph.D. Dissertation. School of Public Policy, University of Maryland.

Sumario: 1. Desigualdad de poder; 2. Agencia y empoderamiento; 3. Democracia y desarrollo; 4. Corrupción; Conclusión; Referencias. 